\title{
In Vitro - In Vivo Correlation (IVIVC): A Strategic Tool in Drug Development
}

\section{Somnath Sakore* and Bhaswat Chakraborty}

Cadila Pharmaceuticals Ltd, Research \& Development, 1389, Trasad Road, Dholka, Ahmedabad 387810, Gujarat, India

\begin{abstract}
In Vitro-In Vivo Correlation (IVIVC) plays a key role in pharmaceutical development of dosage forms. This tool hastens the drug development process and leads to improve the product quality. It is an integral part of the immediate release as well as modified release dosage forms development process. IVIVC is a tool used in quality control for scale up and post-approval changes e.g. to improve formulations or to change production processes \& ultimately to reduce the number of human studies during development of new pharmaceuticals and also to support the biowaivers. This article provides the information on the various guidances, evaluation, validation, BCS application in IVIVC, levels of IVIVC, applications of IVIVC in mapping, novel drug delivery systems and prediction of IVIVC from the dissolution profile characteristics of product.
\end{abstract}

Keywords: IVIVC definitions; Predictions; BCS classification; IVIVC Levels; Applications, Guidance

\begin{abstract}
Abbreviations: IVIVC: In Vitro In Vivo correlation; FDA: Food and Drug Administration; AUC: Area Under Curve; MDT vitro: Mean in vitro Dissolution Time; MRT: Mean Residence Time; BCS: Biopharmaceutical Classification System
\end{abstract}

\section{Introduction}

In vitro in vivo correlations (IVIVC) play a key role in the drug development and optimization of formulation which is certainly a time consuming and expensive process. Formulation optimization requires alteration in formulation, composition, equipments, batch sizes and manufacturing process. If such types of one or more changes are applied to the formulation, the in vivo bioequivalence studies in human may required to be done to prove the similarity of the new formulation which will not only increase the burden of carrying out a number of bioequivalence studies but eventually increase the cost of the optimization process and ultimately marketing of the new formulation. To overcome these problems it is desirable to develop in vitro tests that reflect can bioavailability data. IVIVC can be used in the development of new pharmaceuticals to reduce the number of human studies during the formulation development. Thus, the main objective of an IVIVC is to serve as a surrogate for in vivo bioavailability and to support biowaivers.

IVIVC is a mathematical relationship between in vitro properties of a dosage form with its in vivo performance. The In vitro release data of a dosage form containing the active substance serve as characteristic in vitro property, while the In vivo performance is generally represented by the time course of the plasma concentration of the active substance. These In vitro \& In vivo data are then treated scientifically to determine correlations. For oral dosage forms, the in vitro release is usually measured and considered as dissolution rate. The relationship between the in vitro and in vivo characteristics can be expressed mathematically by a linear or nonlinear correlation. However, the plasma concentration cannot be directly correlated to the in vitro release rate; it has to be converted to the in vivo release or absorption data, either by pharmacokinetic compartment model analysis or by linear system analysis [1].

\section{IVIVC definitions}

\section{United state pharmacopoeia (USP) definition of IVIVC}

The establishment of a rational relationship between a biological property, or a parameter derived from a biological property produced by a dosage form, and a physicochemical property or characteristic of the same dosage form [2].

\section{Food and drug administration (FDA) definition of IVIVC}

An In-vitro in-vivo correlation (IVIVC) has been defined by the Food and Drug Administration (FDA) as "a predictive mathematical model describing the relationship between an in-vitro property of a dosage form and an in-vivo response".

Generally, the In vitro property is the rate or extent of drug dissolution or release while the In vivo response is the plasma drug concentration or amount of drug absorbed. Practically, the purpose of IVIVC is to use drug dissolution results from two or more products to predict similarity or dissimilarity of expected plasma drug concentration (profiles). Before one considers relating in vitro results to in vivo, one has to establish as to how one will establish similarity or dissimilarity of in vivo response i.e. plasma drug concentration profiles. The methodology of establishing similarity or dissimilarity of plasma drug concentrations profile is commonly known as bioequivalence testing. There are very well established guidances and standards available for establishing bioequivalence between drug profiles and products [3].

*Corresponding author: Somnath Sakore, Cadila Pharmaceuticals Ltd, Research \& Development, 1389, Trasad Road, Dholka, Ahmedabad 387810, Gujarat, India, Tel: +91 9879112032; E-mail: drb.chakraborty@cadilapharma.co.in

Received October 06, 2011; Accepted December 31, 2011; Published January 02, 2012

Citation: Sakore S, Chakraborty B (2011) In Vitro-In Vivo Correlation (IVIVC) A Strategic Tool in Drug Development. J Bioequiv Availab S3. doi:10.4172/jbb. S3-001

Copyright: ( 2011 Sakore S, et al. This is an open-access article distributed under the terms of the Creative Commons Attribution License, which permits unrestricted use, distribution, and reproduction in any medium, provided the original author and source are credited. 


\section{Purpose of IVIVC}

\section{Reduction of regulatory burden}

IVIVC can be used as substitute for additional in vivo experiments, under certain conditions.

\section{Optimization of formulation}

The optimization of formulations may require changes in the composition, manufacturing process, equipment, and batch sizes. In order to prove the validity of a new formulation, which is bioequivalent with a target formulation, a considerable amount of efforts is required to study bioequivalence (BE) /bioavailability (BA).

\section{Justification for “therapeutic' product quality}

IVIVC is often adequate for justification of therapeutically meaningful release specifications of the formulation.

\section{Scale up post approval changes (Time and cost saving during the product development)}

Validated IVIVC is also serves as justification for a biowaivers in filings of a Level 3 (or Type II in Europe) variation, either during scaleup or post approval, as well as for line extensions (e.g., different dosage strengths).

\section{IVIVC as surrogate for in vivo bioequivalence and to support biowaivers (Time and cost saving)}

The main purpose of an IVIVC model to utilize in vitro dissolution profiles as a surrogate for in vivo bioequivalence and to support biowaivers.

\section{Levels of Ivivc}

There are four levels of IVIVC that have been described in the FDA guidance, which include levels A, B, C, and multiple C [4]. The concept of correlation level is based upon the ability of the correlation to reflect the complete plasma drug level-time profile which will result from administration of the given dosage form.

\section{Level A correlation}

An IVIVC that correlates the entire in vitro and in vivo profiles has regulatory relevance and is called a Level A Correlation. This level of correlation is the highest category of correlation and represents a point-to-point relationship between in vitro dissolution rate and in vivo input rate of the drug from the dosage form $[3,5]$.

Level A correlation is the most preferred to achieve; since it allows bio waiver for changes in manufacturing site, raw material suppliers, and minor changes in formulation. The purpose of Level A correlation is to define a direct relationship between in vivo data such that measurement of in vitro dissolution rate alone is sufficient to determine the biopharmaceutical rate of the dosage form.

\section{Level B correlation}

A level B IVIVC is based on the principles of statistical moment analysis. In this level of correlation, the mean in vitro dissolution time (MDT vitro) of the product is compared to either mean in vivo residence time (MRT) or the mean in vivo dissolution time (MDTvivo). MRT, MDTvitro and MDTvivo will be defined throughout the manuscript where appropriate [6]. A level B correlation does not uniquely reflect the actual in vivo plasma level curves, also in vitro data from such a correlation could not be used to justify the extremes of quality control standards hence it is least useful for regulatory purposes [5].

\section{Level C correlation}

Level C correlation relates one dissolution time point ( $\mathrm{t} 50 \%, \mathrm{t} 90 \%$, etc.) to one mean pharmacokinetic parameter such as AUC, $\mathrm{t}_{\max }$ or $\mathrm{C}_{\max }$. This is the weakest level of correlation as partial relationship between absorption and dissolution is established since it does not reflect the complete shape of plasma drug concentration time curve, which is the critical factor that defines the performance of a drug product.

Due to its obvious limitations, the usefulness of a Level C correlation is limited in predicting in vivo drug performance. In the early stages of formulation development Level C correlations can be useful when pilot formulations are being selected while waiver of an in vivo bioequivalance study (biowaiver) is generally not possible $[5,6]$.

\section{Multiple level C correlations}

This level refers to the relationship between one or more pharmacokinetic parameters of interest $\left(C_{m a x}, A U C\right.$, or any other suitable parameters) and amount of drug dissolved at several time point of dissolution profile. Multiple point level C correlation may be used to justify a biowaivers provided that the correlation has been established over the entire dissolution profile with one or more pharmacokinetic parameters of interest. A multiple Level C correlation should be based on at least three dissolution time points covering the early, middle, and late stages of the dissolution profile. The development of a level A correlation is also likely, when multiple level $\mathrm{C}$ correlation is achieved at each time point at the same parameter such that the effect on the in vivo performance of any change in dissolution can be assessed $[5,6]$.

\section{Level D correlation}

It is not a formal correlation but it is a semi quantitative (qualitative analysis) and rank order correlation and is not considered useful for regulatory purpose but can be serves as an aid in the development of a formulation or processing procedure [5,7] (Table 1).

\section{IVIVC Models}

The relationship of observed drug concentration-time profiles following administration of a tablet/capsule with drug dissolution and pharmacokinetics may be described graphically as shown in Figure 1.

It is generally assumed that absorption and dissolution have a

\begin{tabular}{|l|l|l|}
\hline Level & In vitro & In vivo \\
\hline A & Dissolution curve & Input (absorption) curves \\
\hline B & $\begin{array}{l}\text { Statistical moments: mean } \\
\text { dissolution time (MDT) }\end{array}$ & $\begin{array}{l}\text { Statistical moments: mean } \\
\text { residence time (MRT), mean } \\
\text { absorption time (MAT), etc }\end{array}$ \\
\hline C & $\begin{array}{l}\text { Disintegration time, time } \\
\text { to have 10\%, 50\%, 90\% } \\
\text { dissolved, dissolution rate, } \\
\text { dissolution efficiency (DE) }\end{array}$ & $\begin{array}{l}\text { Maximum observed concentration } \\
\left(\mathrm{C}_{\text {max }}\right), \text { observed at time }\left(\mathrm{T}_{\text {max }}\right), \\
\text { absorption constant (Ka), Time to have } \\
10,50,90 \% \text { absorbed, AUC } \\
\text { (total or cumulative) }\end{array}$ \\
\hline
\end{tabular}

A: one-to-one relationship between in vitro and in vivo data, e.g., in vitro dissolution vs. in vivo absorption

B: correlation based on statistical moments, e.g., in vitro MDT vs. in vivo MRT or MAT

C: point-to-point relationship between a dissolution and a pharmacokinetic parameter, e.g., in vitro T50\% vs. in vivo T max, Multiple C: relationship between one or several PK parameters and amount dissolved at several time points.

Table 1: Various parameters used in IVIVC depending on the level. 
linear relationship hence dissolution and absorption characteristics of a drug are commonly shown interchangeably. Thus from Figure 2, it is to be noted that one should be able to establish drug profiles with dissolution profiles combined with the pharmacokinetic characteristics of the drug as describe in the example above. This process of obtaining a drug profile from dissolution results is known as convolution. The opposite of this, i.e., obtaining or extracting a dissolution profile from a blood profile, is known as deconvolution Figure 2.

\section{Convolution model}

In the development of convolution model the drug concentrationtime profiles obtained from dissolution results may be evaluated using criteria for in vivo bioavailability/ bioequivalence assessment, based on $\mathrm{C}_{\max }$ and AUC parameters.

In mathematical terminology, dissolution results become an input function and plasma concentrations (e.g. from IV) become a weighting factor or function resulting in an output function representing plasma concentrations for the solid oral product.

Implementation of convolution-based method involves the production of a user-written subroutine for the NONMEM software package, has shown that a convolution-based method based on that of O'Hara et al. [9] produces superior results. Using the NONMEM package, a nonlinear mixed effects model can be fitted to the data with a time-scale model linking the in vitro and in vivo components [10].

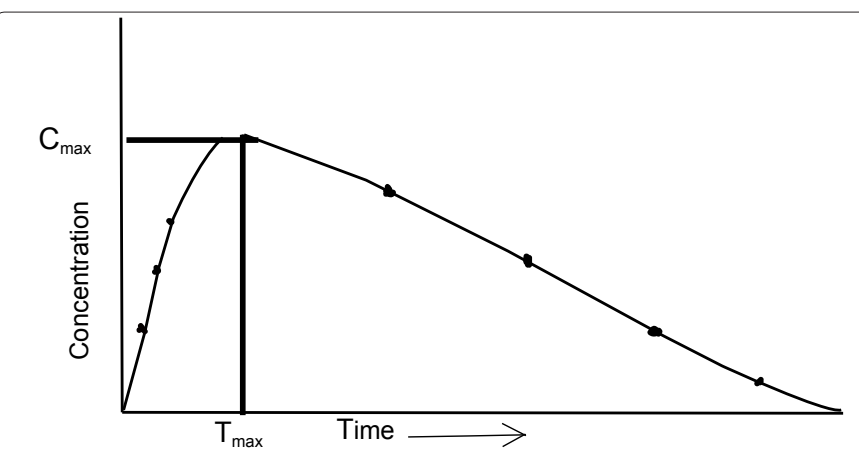

Figure 1: A typical drug concentration (in blood) - time profile reflecting the fate of a drug in the human body following an oral dose (tablet/capsule).
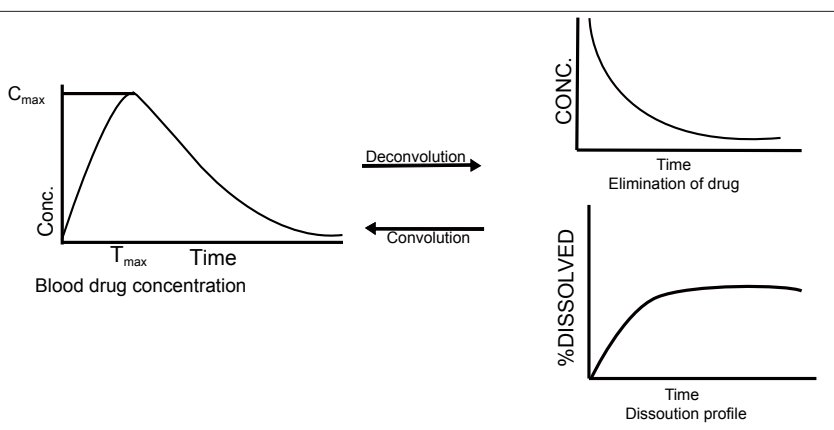

Figure 2: Schematic representation of deconvolution and convolution processes. Convolution is the process of combined effect of dissolution and elimination of drug in the body to reflect blood drug concentration-time profile (right to left). On the other hand, extracting dissolution profiles from blood drug concentration-time profile is known as the deconvolution process (left to right) [8].
It has been demonstrates that the convolution based and differential equation based models can be mathematically equivalent [11]. Software has been developed which implements a differential equation based approach. This method utilises existing NONMEM libraries and is an accurate method of modeling which is far more straightforward for users to implement. This research shows that, when the system being modeled is linear, the use of differential equations will produce results that are practically identical to those obtained from the convolution method.

But is a task that can be time consuming and complex [12]. As a result, this methodology, despite its advantages over the deconvolutionbased approach, is not in widespread use.

Mathematically we can write the convolution as:

$$
C(t)=C_{\delta}(t) F(t)=\int_{0}^{t} C_{\delta}(\tau) F(t-\tau) d \tau
$$

Where, $\mathrm{C}(\mathrm{t})=$ Plasma drug concentrations after oral dose

$\mathrm{C} \delta(\mathrm{t})=$ Plasma concentrations after an IV dose or a dose of oral solution

Upon taking the derivative of $\mathrm{C}(\mathrm{t})$ with respect to time:

$$
C(t)=C_{\delta}(t) F(t)+C_{\delta}(0) \int_{0}^{t} F(\tau) d \tau
$$

When $\mathrm{C} \delta(0)=0$

$C_{\delta}(t) * F(t)$

Advantages of this approach relative to deconvolution-based IVIVC approaches include the following: The relationship between measured quantities (in vitro release and plasma drug concentrations) is modeled directly in a single stage rather than via an indirect two stage approach. The model directly predicts the plasma concentration time course. As a result the modeling focuses on the ability to predict measured quantities (not indirectly calculated quantities such as the cumulative amount absorbed). The results are more readily interpreted in terms of the effect of in vitro release on conventional bioequivalence metrics [5].

\section{Deconvolution model}

Deconvolution is a numerical method used to estimate the time course of drug input using a mathematical model based on the convolution integral.

The deconvolution technique requires the comparison of in vivo dissolution profile which can be obtained from the blood profiles with in vitro dissolution profiles. The observed fraction of the drug absorbed is estimated based on the Wagner-Nelson method. IV, IR or oral solution are attempted as the reference. Then, the pharmacokinetic parameters are estimated using a nonlinear regression tool or obtained from literatures reported previously. Based on the IVIVC model, the predicted fraction of the drug absorbed is calculated from the observed fraction of the drug dissolved. It is the most commonly cited and used method in the literature [10]. However this approach is conceptually difficult to use. For example: (1) Extracting in vivo dissolution data from a blood profile often requires elaborate mathematical and computing expertise. Fitting mathematical models are usually subjective in nature, and thus do not provide an unbiased approach in evaluating in vivo dissolution results/profiles. Even when in vivo dissolution curves are obtained there is no parameter available with associated statistical confidence and physiological relevance, 
which would be used to establish the similarity or dissimilarity of the curves [13]. A more serious limitation of this approach is that it often requires multiple products having potentially different in vivo release characteristics (slow, medium, fast). These products are then used to define experimental conditions (medium, apparatus etc.) for an appropriate dissolution test to reflect their in vivo behavior. This approach is more suited for method/apparatus development as release characteristics of test products are to be known (slow, medium, fast) rather product evaluation [14].

\section{Differential equation based approach}

Another approach, has been proposed is based on systems of differential equations [15]. The use of a differential equation based model could also allow for the possibility of accurately modelling nonlinear systems and further investigation is being carried out into the case where the drug is eliminated by a nonlinear, saturable process. The convolution and deconvolution methods assume that the system being modelled is linear but, in practice, this is not always the case. Work to date has shown that the convolution-based method is superior, but when presented with nonlinear data even this approach will fail. It is expected that, in the nonlinear case, the use of a differential equation based method would lead to more accurate predictions of plasma concentration.

The incorporation of time-scaling in the PDx-IVIVC equation allows this parameter to be estimated directly from the in vivoand vitro release data. As a result, the predictability of an IVIVC model can be evaluated over the entire in vivo time course.Internal predictability of the IVIVC model was assessed using convolution.PDx-IVIVC Model Equation:

$$
x_{\text {vivo }}(t)= \begin{cases}0, & \mathrm{t} \geq 0 . \\ a_{1}+a_{2} x_{\text {vitro }}\left(-b_{1}+b_{2} t\right), & \mathrm{t} \geq 0 .\end{cases}
$$

For orally administered drugs, IVIVC is expected for highly permeable drugs, or drugs under dissolution rate-limiting conditions, which is supported by the Biopharmaceutical Classification System (BCS) [6,16]. For extended-release formulations following oral administration, modified BCS containing the three classes (high aqueous solubility, low aqueous solubility, and variable solubility) is proposed [17].

\section{IVIVC Development}

Any well designed and scientifically sound approach would be acceptable for establishment of an IVIVC. For the development and validation of a IVIVC model, two or three different formulations with different release rates, such as slow, medium, fast should be studied In vitro and In vivo [6].

A number of products with different release rates are usually manufactured by varying the primary rate controlling variable (e.g., the amount of excipient, or a property of the drug substance such as particle size) but within the same qualitative formulation. To develop a discriminative in vitro dissolution method, several method variables together with formulation variables are studied, e.g., different $\mathrm{pH}$ values, dissolution apparatuses and agitation speeds. Essentially at this stage a level A correlation is assumed and the formulation strategy is initiated with the objective of achieving the target in vitro profile. Development of a level A IVIVC model includes several steps.

In context of understanding the applications of IVIVR throughout the product development cycle, it is useful to become familiar with the following terms as they relate to a typical product development cycle for oral extended-release product [5].

An assumed IVIVC is the one that provides the initial guidance and direction for the early formulation development activity. Thus, during step 1 and with a particular desired product, appropriate in vitro targets are established to meet the desired in vivo profile specification. This assumed model can be the subject of revision as prototype formulations are developed and characterized in vivo, with the results often leading to a further cycle of prototype formulation and In vivo characterization.

Out of this product development cycle and In vivo characterization and, of course, extensive in vitro testing is often developed what can be referred to as retrospective IVIVC.

The defined formulation that meets the in vivo specification is employed for Stage 2. At this stage based on a greater understanding and appreciation of defined formulation and its characteristics, a prospective IVIVC is established through a well defined prospective IVIVC study [18,5].

\section{Step 1}

In the first step, the In vivo input profile of the drug from different formulations is calculated from drug concentrations in plasma (Figure 3). The target In vivo profile needs to be first established, based on, if possible, pharmacokinetic/pharmacodynamic models. Certainly, step 1 activity should culminate in a pilot PK study. This is typically a four or five-arm cross-over study. The size of this pilot pharmacokinetic study will vary depending on the inherent variability of the drug itself but typically range from 6 to 10 subjects [5]. The results of this pilot PK study provide the basis for establishing what has been referred to as a retrospective IVIVC. To separate drug input from drug distribution and elimination, model-dependent approaches, such as WagnerNelson and Loo-Riegelman, or model independent procedures, based on numerical deconvolution, may be utilised $[19,20,21]$. In step 1 , the parameters that describe drug input rate, drug distribution and/or elimination are determined. In the model dependent approaches, the distribution and elimination rate constants describe pharmacokinetics after absorption. In the numerical deconvolution approach, the drug unit impulse response function describes distribution and elimination phases, respectively. The physicochemical characteristics of the drug substance itself, in relevance to formulation approach and dissolution at distal sites in the gastro-intestinal tract, need to be taken into account. Based on this information a priori in vitro methods are usually then developed and a theoretical in vitro target is established, which should achieve the desired absorption profile [5,18].

\section{Step 2}

By this phase of the development process, a defined formulation that meets the In vivo targets has been achieved. Extensive In vitro characterization is again performed across $\mathrm{pH}$, media and apparatus, along with the consideration of results of stage 1 . This leads to execution of a prospective IVIVC study. The IVIVC is developed and defined after an analysis of the result of that prospective in vivo study. It can often involved further in vitro method development in the context of the observed results, but clearly with the objective of establishing a definitive IVIVC. In this step, the relationship between 
Citation: Sakore S, Chakraborty B (2011) In Vitro-In Vivo Correlation (IVIVC): A Strategic Tool in Drug Development. J Bioequiv Availab S3. doi:10.4172/jbb.S3-001

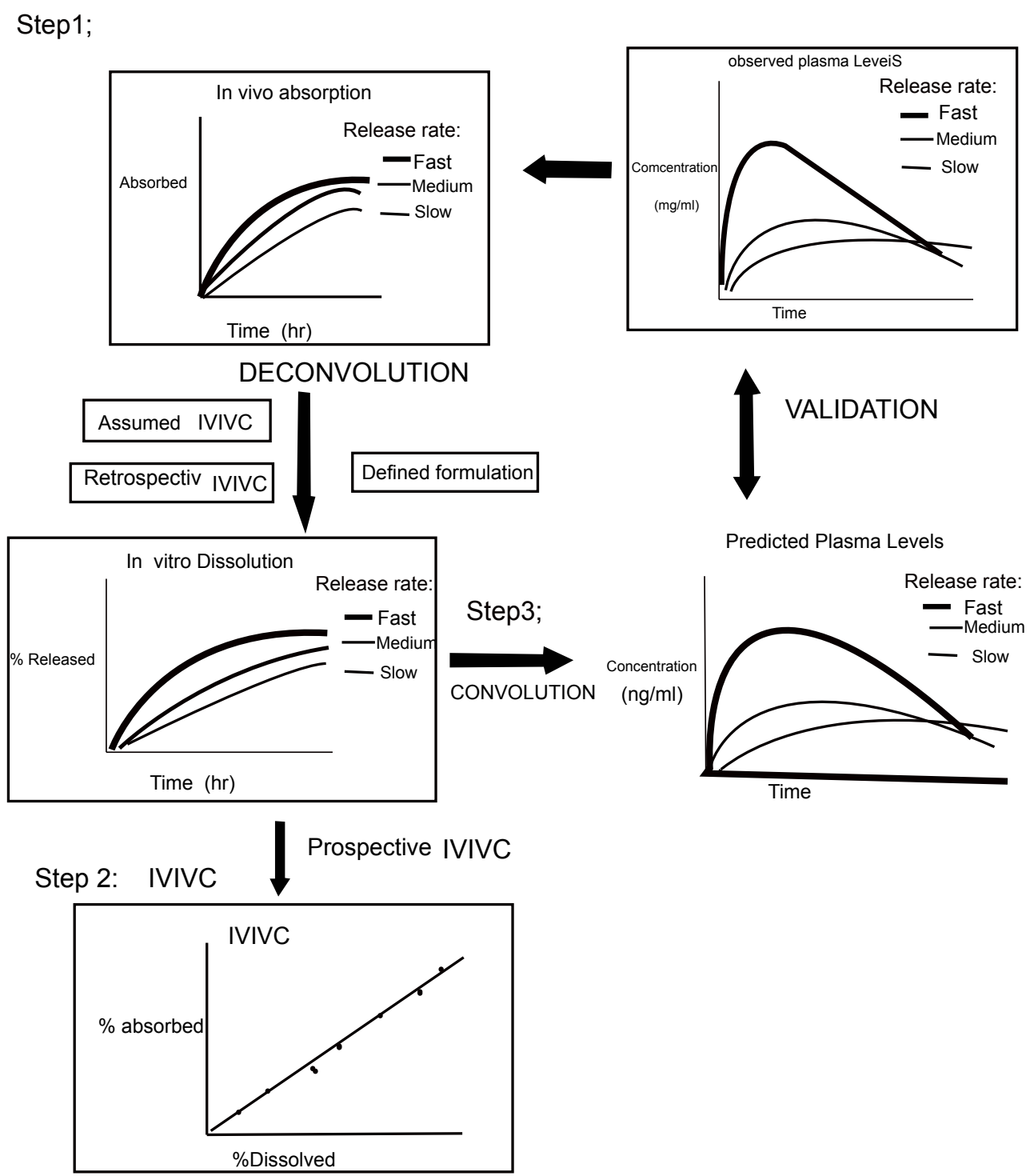

Figure 3: Development of IVIVC with validation.

in vitro dissolution and the in vivo drug input profile is determined (Figure 3). Either a linear or nonlinear relationship may be found. In some cases, time-scaling of in vitro data must be used, because In vitro dissolution and In vivo input may follow the same kinetics but still have different time-scales [6,22]. The time-scaling factor should be the same for all formulations if an IVIVC at level A is sought. During the early stages of correlation development, dissolution conditions may be altered to attempt to develop a 1-to-1 correlation between the in vitro dissolution profile and the In vivo dissolution profile. This work should also result in the definitive in vitro method that has been shown to be correlated with in vivo performance and sensitive to the specific formulation variables.

\section{Step 3}

In this phase plasma drug concentration profiles are predicted and compared to the observed time courses for different formulations (Figure 3). To generate predicted time courses, the drug input profile is predicted based on In vitro dissolution data and the In vitro-In vivo relationship generated in step 2 . In the convolution process, the predicted drug input and parameters describing drug distribution and/ or elimination phases are combined in order to get predicted time courses. This procedure, which includes steps 1-3, is called two-stage deconvolution. Alternatively, a drug input profile based on in vitro dissolution data can be solved together with parameters describing systemic pharmacokinetics, i.e. distribution and elimination. This approach is called direct convolution.

Different IVIVC model are used as a tool for formulation development and evaluation of immediate and extended release dosage forms for setting a dissolution specification and as a surrogate for bioequivalence testing.

As a result, considerable effort goes into their development and the main outcome is "the ability to predict, accurately and precisely, expected bioavailability characteristics for an extended release (ER) drug product from dissolution profile characteristics [10]. 
Once the IVIVC is established and defined it can be then used to guide the final cycle of formulation and process optimization program statistically based experimental design studies looking at critical formulation and process variables. This information can also be used into the activities of scale-up, pivotal batch manufacture, and process validation culminating in registration, approval and subsequent postapproval scale-up and other changes. Thus rather than viewing the IVIVC as a single exercise at a given point in a development program, one should view it as a parallel development in itself starting at the initial assumed level and being built on and modified through experience and leading ultimately to a prospective IVIVC".

\section{Validation of IVIVC Model}

\section{Evaluation of predictability of IVIVC}

Prediction errors are estimated for $C_{\max }$ and AUC to determine the validity of the correlation.

Various approaches of are used to estimate the magnitude of the error in predicting the in vivo bioavailability results from in vitro dissolution data.

\section{Predictability of correlation}

The objective of IVIVC evaluation is to estimate the magnitude of the error in predicting the in vivo bioavailability results from in vitro dissolution data. This objective should guide the choice and interpretation of evaluation methods. Any appropriate approach related to this objective may be used for evaluation of predictability $[5,23]$. It can be calculated by Prediction error that is the error in prediction of in vivo property from in vitro property of drug product (Figure 3).

Depending on the intended application of an IVIVC and the therapeutic index of the drug, evaluation of prediction error internally and/or externally may be appropriate [24].

\section{Internal predictability}

Evaluation of internal predictability is based on the initial data used to define the IVIVC model. Internal predictability is applied to IVIVC established using formulations with three or more release rates for nonnarrow therapeutic index drugs exhibiting conclusive prediction error. If two formulations with different release rates are used to develop IVIVC, then the application of IVIVC would be limited to specified categories. The bioavailability $\left(\mathrm{C}_{\max }, \mathrm{t}_{\max } / \mathrm{AUC}\right)$ of formulation that is used in development of IVIVC is predicted from its in vitro property using IVIVC. Comparison between predicted bioavailability and observed bioavailability is done and \% P.E is calculated. According to FDA guidelines, the average absolute \%P.E should be below $10 \%$ and $\%$ P.E for individual formulation should be below $15 \%$ for establishment of IVIVC.

Under these circumstances, for complete evaluation and subsequent full application of the IVIVC, prediction of error externally is recommended [23].

\section{Acceptance criteria}

According to FDA guidance

1) $\leq 15 \%$ for absolute prediction error (\%P.E.) of each formulation.

2) $\leq 10 \%$ for mean absolute prediction error (\%P.E.).

\section{External predictability}

Most important when using an IVIVC as a surrogate for bioequivalence is confidence that the IVIVC can predict in vivo performance of subsequent lots of the drug product. Therefore, it may be important to establish the external predictability of the IVIVC.

Evaluation of external predictability is based on additional test data sets [5]. External predictability evaluation is not necessary unless the drug is a narrow therapeutic index, or only two release rates were used to develop the IVIVC, or, if the internal predictability criteria are not met i.e. prediction error internally is inconclusive $[4,23]$. The predicted bioavailability is compared with known bioavailability and \% P.E is calculated. The prediction error for external validation should be below $10 \%$ whereas prediction error between $10-20 \%$ indicates inconclusive predictability and need of further study using additional data set [24].

The $\%$ prediction error can be calculated by the following equation:

\section{Prediction error}

For $\mathbf{C}_{\text {max }}$

\%Prediction error $($ P.E. $)=\frac{\left(C_{\max } \text { observed }-C_{\max } \text { predicted }\right)}{C_{\max } \text { observed }} \times 100$ (5)
For AUC:

$\%$ Prediction error $($ P.E. $)=\frac{(\text { AUC } \text { observed }- \text { AUC } \text { predicted })}{\text { AUC } \text { observed }} \times 100(6)$

\section{Limitation of predictability metrics}

Metrics used to evaluate the predictability is described simply the prediction error (\%P.E.) for only two $\mathrm{PK}$ parameters i.e. $\mathrm{C}_{\max }$ and AUC. $\mathrm{E}_{\max }$ predicted with IVIVC model represents the maximum of the mean plasma profiles but is compared with the mean $\mathrm{C}_{\text {max }}$ observed calculated as the average of individual profile at different $\mathrm{T}_{\max }$. But $\mathrm{T}_{\max }$ is not included in predictability metrics.

\section{Factors to be Consider in Developing a Correlation Biopharmaceutics classification system (BCS)}

Biopharmaceutics Classification System (BCS) is a fundamental guideline for determining the conditions under which in-vitro, in-vivo correlations are expected [25]. It is also used as a tool for developing the in-vitro dissolution specification.

The classification is based on the drug dissolution and absorption model, which identifies the key parameters controlling drug absorption as a set of dimensionless numbers: the Absorption number, the Dissolution number and the Dose number [25-27].

The Absorption number is the ratio of the mean residence time to the absorption time.

$A_{n}=T_{r e s} / T_{a b s}+\left(\pi R^{2} L / Q\right) /\left(R / P_{e f f}\right)$

The Dissolution number is a ratio of mean residence time to mean dissolution time given as equation 2

$$
D_{n}=T_{\text {res }} / T_{\text {diss }}+\left(\pi R^{2} L / Q\right) /\left(p r_{0}^{2} / 3 D C_{s}^{\min }\right)
$$

The Dose number is the mass divided by an uptake volume of 250 $\mathrm{ml}$ and the drug's solubility as Equation 3

$$
D_{o}=\text { Dose } /\left(V_{o} / C_{s}^{\text {min }}\right)
$$

The mean residence time here is the average of the residence time 
in the stomach, small intestine and the colon. The fraction of dose absorbed then can be predicted based on these three parameters. For example, Absorption number 10 means that the permeation across the intestinal membrane is 10 times faster than the transit through the small intestine indicating $100 \%$ drug absorbed.

In the BCS, a drug is classified in one of four classes based solely on its solubility and intestinal permeability [27].

A biopharmaceutic drug classification scheme for correlating in vitro drug product dissolution and in vivo bioavailability is proposed based on recognizing that drug dissolution and gastrointestinal permeability are the fundamental parameters controlling rate and extent of drug absorption. This classification system was devised by Amidon et al. [27].

The drugs are divided into high/low-solubility and permeability classes. Currently, BCS guidelines are provided by USFDA, WHO, and EMEA (European Medicines Academy)

Class I: HIGH solubility / High permeability,

Class II: LOW solubility / High permeability,

Class III: HIGH solubility / LOW permeability,

Class IV: LOW solubility / LOW permeability.

\section{Class I: High solubility- high permeability drugs}

In case of class I, drugs (such as metoprolol) is well absorbed (though its systemic availability may be low due to first pass extraction/ metabolism) and the rate limiting step to drug absorption is drug dissolution or gastric emptying if dissolution is very rapid. The dissolution specification immediate release (IR) dosage forms of perhaps $85 \%$ dissolved in less than $15 \mathrm{~min}$. May insure bioequivalence. To insure bioavailability for this case, the dissolution profile must be well defined and reproducible. [5,27].

\section{Class II: Low solubility- high permeability drugs}

This is the class of drugs (such as phenytoin) for which the dissolution profile must be most clearly defined and reproducible. More precisely this is the case where absorption number, (An) is high and Dissolution number (Dn) is low. Drug dissolution in vivo is then the rate controlling step in drug absorption and absorption is usually slower than for class I [28-31].

\section{Class III: High solubility-low permeability drugs}

For this class of drugs (such as cimetidine) Permeability is the rate controlling step in drug absorption. While the dissolution profile must be well defined, the simplification in dissolution specification as in Class I is applicable for immediate release dosage forms where drug input to the intestine is gastric emptying rate controlled.. Both rate and extent of drug absorption may be highly variable for this class of drugs, but id dissolution is fast i.e. $85 \%$ dissolved in less than $15 \mathrm{~min}$, this variation will be due to the variable gastrointestinal transit, luminal contents , and membrane permeability rather than dosage form factors [5].

\section{Class IV: Low solubility-low permeability drugs}

This class of drugs present significant problems for effective oral delivery. The number of drugs that fall in this class will depend on the precise limits used from the permeability and solubility classification.

\section{Applications}

This concept underlying the BCS published finally led to introducing the possibility of waiving in vivo bioequivalence (BE) studies in favor of specific comparative in vitro testing to conclude $\mathrm{BE}$ of oral immediate release (IR) products with systemic actions [32].

In terms of $\mathrm{BE}$, it is assumed that highly permeable, highly soluble drugs housed in rapidly dissolving drug products will be bioequivalent and that, unless major changes are made to the formulation, dissolution data can be used as a surrogate for pharmacokinetic data to demonstrate $\mathrm{BE}$ of two drug products. The BCS thus enables manufacturers to reduce the cost of approving scale-up and post approval changes to certain oral drug products without compromising public safety interests [33].

It is a drug-development tool that allows estimation of the contributions of three major factors, dissolution, solubility and intestinal permeability that affect oral drug absorption from IR solid oral dosage forms. It was first introduced into regulatory decisionmaking process in the guidance document on immediate release solid oral dosage forms: Scale-up and post approval changes [2]. BCS system is an indicator of developing a predictive IVIVC and also examined the importance of drug dissolution and permeability on IVIVC validity (Table 2).

The establishment of correlation needs, as described in the FDA or USP definitions, to use various parameters summarized in following table: Waiver of in vivo BE studies

\begin{tabular}{|l|l|l|l|}
\hline Class & Solubility & Permeability & IVIVC correlation for IR Products \\
\hline I & High & High & $\begin{array}{l}\text { IVIVC correlation if dissolution rate is slower } \\
\text { than gastric emptying rate, otherwise limited } \\
\text { or no correlation }\end{array}$ \\
\hline II & Low & High & $\begin{array}{l}\text { IVIVC correlation expected if in in vitro } \\
\text { dissolution rate is similar to in vivo dissolution } \\
\text { rate unless dose is very high }\end{array}$ \\
\hline III & High & Low & $\begin{array}{l}\text { Absorption [permeability] is rate determining } \\
\text { and limited or no IVIV correlation with } \\
\text { dissolution rate. }\end{array}$ \\
\hline IV & Low & Low & Limited or no IVIV correlation expected. \\
\hline
\end{tabular}

Table 2: IVIV correlation expectation for immediate release product based on biopharmaceutic class.

\begin{tabular}{|c|c|c|c|}
\hline \multicolumn{4}{|c|}{$\begin{array}{l}\text { Biopharmaceutics Drug Classification } \\
\text { for Extended Release Drug Products ** }\end{array}$} \\
\hline Class & Solubility & Permeability & IVIVC \\
\hline IA & $\begin{array}{l}\text { High \& Site } \\
\text { Independent }\end{array}$ & High \& Site Independent & $\begin{array}{l}\text { IVIVC Level A } \\
\text { expected }\end{array}$ \\
\hline IB & $\begin{array}{l}\text { High \& Site } \\
\text { Independent }\end{array}$ & $\begin{array}{l}\text { Dependent on site \& Narrow } \\
\text { Absorption Window }\end{array}$ & $\begin{array}{l}\text { IVIVC Level } \\
\text { C expected }\end{array}$ \\
\hline Ila & $\begin{array}{l}\text { Low \& Site } \\
\text { Independent }\end{array}$ & High \& Site Independent & $\begin{array}{l}\text { IVIVC Level A } \\
\text { expected }\end{array}$ \\
\hline llb & $\begin{array}{l}\text { Low \& Site } \\
\text { Independent }\end{array}$ & $\begin{array}{l}\text { Dependent on site \& Narrow } \\
\text { Absorption Window }\end{array}$ & $\begin{array}{l}\text { Little or no } \\
\text { IVIVC }\end{array}$ \\
\hline $\begin{array}{l}\text { Va: } \\
\text { Acidic }\end{array}$ & Variable & Variable & $\begin{array}{l}\text { Little or no } \\
\text { IVIVC }\end{array}$ \\
\hline $\begin{array}{l}\text { Vb: } \\
\text { basic }\end{array}$ & Variable & Variable & $\begin{array}{l}\text { IVIVC Level A } \\
\text { expected. }\end{array}$ \\
\hline
\end{tabular}

Table 3: Biopharmaceutics Drug Classification for Extended Release Drug Products. 


\begin{tabular}{|l|l|l|}
\hline BCS Class & Examples & Drug delivery technology \\
\hline Class I & $\begin{array}{l}\text { Metoprolol, Diltizem, Verapamil, Propranolol, } \\
\text { Acyclovir, Atropine, verapamil. }\end{array}$ & $\begin{array}{l}\text { Macrocap, Micropump, MODAS (Multiporous oral drug absorption system), SCOT (Single } \\
\text { composition osmotic tablet system), and SPDS (Stabilized pellet delivery system) [28,30,31]. }\end{array}$ \\
\hline Class II & $\begin{array}{l}\text { Phenytoin, Danazole, Ketokonazole, Mefenamic } \\
\text { acid, Tacrolimus, Piroxicam, griseofulvine, } \\
\text { Warfarin, }\end{array}$ & $\begin{array}{l}\text { Micronization, stabilization of high-energy states (including lyophilized fast-melt systems), use of } \\
\text { surfactants, emulsion or microemulsion systems, solid dispersion and use of complexing agent such } \\
\text { as cyclodextrins.e.g nanosuspension and nanocrystals are treated as hopeful means of increasing } \\
\text { solubility and BA of poorly water-soluble active ingredients [28,30,31]. }\end{array}$ \\
\hline Class III & Cimetidine, Neomycin, ranitidine, Amoxycillin, & $\begin{array}{l}\text { Oral vaccine system, Gastric retention system, High-Frequency Capsule and Telemetric Capsule } \\
{[28,30,31] .}\end{array}$ \\
\hline Class IV & $\begin{array}{l}\text { Cyclosporin A, Furosemide, Ritonavir, } \\
\text { Saquinavir andTaxol. }\end{array}$ & $\begin{array}{l}\text { The class IV drugs present a major challange for the development of drug delivery systems due to } \\
\text { their poor solubility and permeability characteristics. These are administered by parenteral route } \\
\text { with the formulation containing solubility enhancers [ 28,30,31]. }\end{array}$ \\
\hline
\end{tabular}

Table 4: BCS class and drug delivery technology.

based on BCS Recommended for a solid oral Test product that exhibit rapid ( $85 \%$ in $30 \mathrm{~min}$ ) and similar in vitro dissolution under specified conditions to an approved Reference product when the following conditions are satisfied (Table 3,4):

- Products are pharmaceutical equivalent

- Drug substance is highly soluble and highly permeable and is not considered have a narrow therapeutic range

- Excipients used are not likely to effect drug absorption

\section{In vitro dissolution}

Dissolution plays the important role in the formulation development as an obvious stage in IVIVC development when the dissolution is not influenced by factors such as $\mathrm{pH}$, surfactants, osmotic pressure, mixing intensity, enzyme, ionic strength. Drug absorption from a solid dosage form following oral administration depends on the release of the drug substance from the drug product, the dissolution or solubilization of the drug under physiological conditions, and the permeability across the gastrointestinal tract. The purpose of the invitro dissolution studies in the early stage of drug development is to select the optimum formulation, evaluate the active ingredient and excipients, and assess any minor changes for drug products. During the early stages of correlation development, dissolution conditions may be altered to attempt to develop a one-to-one correlation between the in vitro dissolution profile and the in vivo dissolution profile [5]. For the IVIVC perspective, dissolution is proposed to be a surrogate of drug bioavailability. Thus, dissolution standard may be necessary for the in-vivo waiver [26]. The dissolution methodology, which is able to discriminate between the study formulations and which best, reflects the in vivo behavior would be selected. Once a discriminating system is developed, dissolution conditions should be the same for all formulations tested in the biostudy for development of the correlation and should be fixed before further steps towards correlation evaluation are undertaken [34]. The types of dissolution apparatus used as per USP recommended in the FDA guidance especially, for modified release dosage form are specified by the USP and are:

[1] Rotating basket,

[2] Paddle method,

[3] Reciprocating cylinder,

[4] Flow through cell,

Other dissolution methodologies may be used, however, the first four are preferred, especially the basket and paddle. But primarily it is recommended to start with the basket or paddle method prior to using the others [26].

The in vitro dissolution release of a formulation can be modified to facilitate the correlation development. Changing dissolution testing conditions such as stirring speed, choice of apparatus, $\mathrm{pH}$ of the medium, and temperature may alter the dissolution profile.

As previously described, appropriate dissolution testing conditions should be selected so that the formulation behaves in the same manner as the in vivo dissolution.

For an appropriate dissolution test, in general and in particular for developing IVIVC, one requires to conduct the test selecting experimental conditions to simulate an in vivo environment as closely as possible. Commonly the following experimental conditions should be considered in this regard.

A common dissolution medium is dearated water, simulated gastric fluid ( $\mathrm{pH} 1.2$ ), or intestinal fluid ( $\mathrm{pH} 6.8$ or 7.4) without enzyme, and buffers with a pH range of 4.5 to 7.5 and be maintained at $37^{\circ} \mathrm{C}$. For sparingly water-soluble drugs, use of surfactants in the dissolution medium is recommended [34,35]. A simple aqueous dissolution media is also recommended for BCS Class I drug as this type of drug exhibits lack of influence of dissolution medium properties [5,36]. Water and simulated gastric fluid then are the default mediums for most of the Class I drugs. A typical medium volume is 500 to $1000 \mathrm{ml}$.

1. Frequent samples $(8-10)$ should be withdrawn to obtain a smooth dissolution profile leading to complete dissolution within the dosing interval of the test product in humans.

2. The normal test duration for immediate release is 15 to 60 minutes with a single time point. For example, BCS class I recommend 15 minutes. Additionally, two time points may be required for the BCS class II at 15 minutes and the other time at which $85 \%$ of the drug is dissolved [36].

3. In contrast, in vitro dissolution tests for a modified release dosage form require at least three time points to characterize the drug release. The first sampling time (1-2 hours or $20-30 \%$ drug release) is chosen to check dose-dumping potential. The intermediate time point has to be around 50\% drug release in order to define the in vitro release profile.

4. The dissolution medium should not be de-aerated. Preference should be given that the medium be equilibrated at $37^{\circ} \mathrm{C}$ with dissolved air/gasses, particularly for IVIVC studies.

5. An apparatus should be selected to have an appropriate 
mechanism to provide thorough but gentle mixing and stirring for an efficient product/medium interaction. Use of sinkers may be avoided as these often alter the dissolution characteristics of the test products. Paddle and basket apparatuses are known for their inefficient stirring and mixing, thus their use should be critically evaluated before use for IVIVC studies.

6. The last time point is to define essentially complete drug release. The dissolution limit should be at least $80 \%$ drug release. Further justification as well as 24 hours test duration are required if the percent drug release is less than 80 [34,37].

7. If the dissolution results are not as expected, then the product/ formulation should be modified to obtain the desired/expected release characteristics of the product. However, altering experimental conditions such as medium, apparatus, rpm etc. should be avoided as these are generally linked to GI physiology which remains the same for test to test or product to product. Obtaining dissolution results by altering testing (experimental) conditions may void the test for IVIVC purposes.

Once the discriminatory system is established, dissolution testing conditions should be fixed for all formulations tested for development of the correlation [6]. A dissolution profile of percentage or fraction of drug dissolved versus time then can be determined.

Comparison between dissolution profiles could be achieved using a difference factor $\left(f_{1}\right)$ and a similarity factor $\left(f_{2}\right)$ which originates from simple model independent approach. The difference factor calculates the percent difference between the two curves at each time point and is a measurement of the relative error between the two curves:

$$
f_{1}=\left\{\left[\sum_{t=1}^{n}\left|R_{t}-T_{t}\right|\right] /\left[\sum_{r=1}^{n} \mid R_{t}\right] * 100\right\}
$$

Where, $\mathrm{n}$ is the number of time points, $\mathrm{Rt}$ is the dissolution value of the reference batch at time $t$, and $\mathrm{Tt}$ is the dissolution value of the test batch at time $t$. The similarity factor is a logarithmic reciprocal square root transformation of the sum squared error and is a measurement of the similarity in the percent dissolution between the two curves

$$
f_{2}=50 * \log \left\{\left[1+(1 / n) \sum_{t=1}^{n}\left(R_{t}-T_{t}\right)^{2}\right]^{-0.5} * 100\right\}
$$

Generally, f1 values up to $15(0-15)$ and $\mathrm{f} 2$ values greater than 50 (50-100) ensure sameness or equivalence of the two curves. The mean in vitro dissolution time (MDTvitro) is the mean time for the drug to dissolve under in vitro dissolution conditions. This is calculated using the equation 6 :

$$
M D T_{\text {vitro }}=\int_{0}^{\infty}\left(M_{\infty}-M(t)\right) d t / M_{\infty}
$$

For the IVIVC development, the dissolution profiles of at least 12 individual dosage units from each lot should be determined. The coefficient of variation (CV) for mean dissolution profiles of a single batch should be less than 10\%. Since dissolution apparatuses tend to become less discriminative when operated at faster speeds, lower stirring speeds should be evaluated and an appropriate speed chosen in accordance with the test data. Using the basket method the common agitation is $50-100 \mathrm{rpm}$; with the paddle method, it is $50-75 \mathrm{rpm}$ and 25 rpm for suspension [5].

\section{In vivo absorption (Bioavailability studies)}

The FDA requires in vivo bioavailability studies to be conducted for a New Drug Application (NDA). A bioavailability study should be performed to characterize the plasma concentration versus time profile for each of the formulation. These studies for the development of IVIVC should be performed in young healthy male adult volunteers under some restrictive conditions such as fasting, non-smoking, and no intake of other medications. In prior acceptable data sets, the number of subjects has ranged from 6 to 36. Although crossover studies are preferred, parallel studies or cross-study analyses may be acceptable. The latter may involve normalization with a common reference treatment. The drug is usually given in a crossover fashion with a washout period of at least five half-lives.

The bioavailability study can be assessed via plasma or urine data using the following parameters: (I) area under the plasma time curve (AUC), or the cumulative amount of drug excreted in urine (Du), (II) maximum concentration $\left(\mathrm{C}_{\max }\right)$, or rate of drug excretion in urine $(\mathrm{dDu} / \mathrm{dt})$, and (III) a time of maximum concentration $\left(\mathrm{T}_{\max }\right)$.

Several approaches can be used for determining the In vivo absorption. Wagner-Nelson, Loo-Riegelman, and numerical deconvolution are such methods [2,37]. Wagner Nelson and LooRiegelman are both model dependent methods in which the former is used for a one-compartment model and the latter is for multicompartment system.

The Wagner Nelson method is less complicated than the LooRiegelman as there is no requirement for intravenous data. However, misinterpretation on the terminal phase of the plasma profile may be possible in the occurrence of a flip flop phenomenon in which the rate of absorption is slower than the rate of elimination.

\section{Application of An IVIVC}

\section{Application in drug delivery system}

Various rate controlling technologies are used as the basis for Modified release dosage forms e.g. Diffusion-dissolution, matrix retardation, osmosis, etc. to control, and prolong the release of drugs, for the administration by oral or parenteral route $[24,38]$.

The novel drug delivery systems have been developed such as OROS, liposomes, niosomes, pharmacosomes, microspheres, nanoparticles, implants, in situ gelling system, organogels, transdermal drug delivery systems, parenteral depots, etc. as a substitute for conventional dosage forms. The obvious objective of these dosage forms is to achieve zero-order, long term, pulsatile, or "on demand" delivery. Major applications of IVIVC related to oral drug delivery and a few issues related to the development of IVIVC models for parenteral drug delivery are addressed herewith [39].

\section{In early stages of drug delivery technology development}

The most crucial stage in the drug development is drug candidate selection. Such selection is primarily based on the drug "developability" criteria, which include physicochemical properties of the drug and the results obtained from preformulation, preliminary studies involving several in vitro systems and in vivo animal models, which address efficacy and toxicity issues [24,40]. During this stage, IVIVC (exploring the relationship between in vitro and in vivo properties) of the drug in animal models provide an idea about the feasibility of the drug delivery system for a given drug candidates. In such correlations, study designs including study of more than one formulation of the modified-release 
dosage forms and a rank order of release (fast/slow) of the formulations should be incorporated. Even though the formulations and methods used at this stage are not optimal, they promise better design and development efforts in the future.

\section{Formulation assessment: In vitro dissolution}

A suitable dissolution method that is capable of distinguishing the performance of formulations with different release rates in vitro and in vivo is an important tool in product development. Depending on the nature of the correlation, further changes to the dissolution method can be made. When the discriminatory in vitro method is validated, further formulation development can be relied on the in vitro dissolution only.

\section{Dissolution specifications}

Modified-release dosage forms typically require dissolution testing over multiple time points, and IVIVC plays an important role in setting these specifications [24,39]. Specification time points are usually chosen in the early, middle, and late stages of the dissolution profiles. In the absence of an IVIVC, the range of the dissolution specification rarely exceeds $10 \%$ of the dissolution of the pivotal clinical batch. However, in the presence of IVIVC, wider specifications may be applicable based on the predicted concentration-time profiles of test batches being bioequivalent to the reference batch.

The process of setting dissolution specifications in the presence of an IVIVC starts by obtaining the reference (pivotal clinical batch) dissolution profile. The dissolution of batches with different dissolution properties (slowest and fastest batches included) should be used along with the IVIVC model, and prediction of the concentration time profiles should be made using an appropriate convolution method. Specifications should optimally be established such that all batches with dissolution profiles between the fastest and slowest batches are bioequivalent and less optimally bioequivalent to the reference batch. The above exercise in achieving the widest possible dissolution specification allows majority of batches to pass and is possible only if a valid Level A model is available [24].

\section{Future biowaivers}

Frequently, drug development requires changes in formulations due to a variety of reasons, such as unexpected problems in stability, development, availability of better materials, better processing results, etc. Having an established IVIVC can help avoid bioequivalence studies by using the dissolution profile from the changed formulation, and subsequently predicting the in vivo concentration-time profile $[24,41]$.

This predicted profile could act as a surrogate of the in vivo bioequivalence study. This has enormous cost-saving benefit in the form of reduced drug development spending and speedy implementation of post-approval changes. The nature of post-approval changes could range from minor (such as a change in non release-controlling excipient) to major (such as site change, equipment change, or change in method of manufacture, etc) $[24,42]$.

\section{IVIVC - Parenteral drug delivery}

IVIVC can be developed and applied to parenteral dosage forms, such as controlled-release particulate systems, depot system, implants, etc, that are either injected or implanted. However, there are relatively fewer successes in the development of IVIVC for such dosage forms, which could be due to several reasons, a few of which are discussed further. Sophisticated modeling techniques are needed to correlate the in vitro and in vivo data, in case of burst release which is unpredictable and unavoidable $[24,43]$.

Potent Drugs \& Chronic Therapy - In general, several parenteral drug delivery systems are developed for potent drugs (eg, hormones, growth factors, antibiotics, etc) and for long-term delivery (anywhere from a day to a few weeks to months). In such instances, to establish a good IVIVC model, the drug concentrations should be monitored in the tissue fluids at the site of administration by techniques such as microdialysis, and then the correlation should be established to the in vitro release.

\section{Biowaivers}

Validated IVIVC is applicable to serve as justification for a biowaiver in filings of a Level 3 (or Type II in Europe) variation, either during scale-up or post approval, as well as for line extensions (e.g., different dosage strengths). A biowaiver will only be granted if the prediction of the in vivo performance of the product with the modified in vitro release rate remains bioequivalent with the originally tested product (i.e., the new dissolution rate remains within the IVIVC based biorelevant corridor).

The FDA guidance outlines five categories of biowaivers: 1) biowaivers without an IVIVC, 2) biowaivers using an IVIVC: nonnarrow therapeutic index drugs, 3 ) biowaivers using an

IVIVC: narrow therapeutic index drugs, 4) biowaivers when in vitro dissolution is independent of dissolution test conditions and 5) situations for which an IVIVC is not recommended for biowaivers Biowaivers may be granted for manufacturing site changes, equipment changes, manufacturing process changes, and formulation composition changes according to a predictive and reliable IVIVC. The changes may range from minor changes that are not significant to alter product performance to major ones where an IVIVC is not sufficient to justify the change for regulatory decision $[4,24]$.

\section{Establishment of dissolution specifications}

It is relatively easy to establish a multipoint dissolution specification for modified-release dosage forms. The dissolution behavior of the biobatch maybe used to define the amount to be released at each time point. However, the difficulty arises in the variation to be allowed around each time point [37]. The FDA guidance describes the procedures of setting dissolution specifications in cases of level A, multiple levels $\mathrm{C}$, and level C correlation and where there is no IVIV correlation. Once an IVIVC developed, IVIVC should be used to set specifications in such a way that the fastest and lowest release rates allowed by the upper and lower dissolution specifications result in a maximum difference of $20 \%$ in the predicted $\mathrm{C}_{\max }$ and AUC. Predicted plasma concentration and consequent $\mathrm{AUC}$ and $\mathrm{C}_{\max }$ could be calculated using convolution or any other appropriate modeling techniques [24]. In the case of multiple level $\mathrm{C}$ correlation, the last time point should be the time point where at least $80 \%$ of drug has dissolved. For level C correlation, reasonable deviations from $\pm 10 \%$ may be acceptable if the range at any time point does not exceed $25 \%$. When there is no IVIVC, the tolerance limits may be derived from the spread of in vitro dissolution data of batches with demonstrated acceptable in vivo performance (biobatch) or by demonstrating bioequivalence between batches at the proposed upper and lower limit of the dissolution range (the so called side batch 
concept). Variability in release at each time point is recommended not to exceed a total numerical difference of $\pm 10 \%$ (a total of $20 \%$ ) or less of the labeled claim. In certain cases, deviations from this criterion can be acceptable up to a maximum range of $25 \%$. Beyond this range, the specification should be supported by bioequivalence studies [43].

\section{Mapping}

Mapping is a process which relates Critical Manufacturing Variables (CMV), including formulation, processes, and equipment variables that can significantly affect drug release from the product. The mapping process defines boundaries of in vitro dissolution profiles on the basis of acceptable bioequivalency criteria. The optimum goal is to develop product specifications that will ensure bioequivalence of future batches prepared within the limits of acceptable dissolution specifications. Dissolution specifications based on mapping would increase the credibility of dissolution as a bioequivalency surrogate marker and will provide continuous assurance and predictability of the product performance [5].

\section{Some Limitations in the IVIVC Arising from the In Vivo Data}

Could easily be understood:

1. More than one dosage form is needed and if possible intravenous or solution is essential to calculate deconvolution.

2. Pharmacokinetics and absorption of the drug should be "linear." If the pharmacokinetic processes are dependent on the fraction of dose reaching the systemic blood flow (or of the dose administered) or on the rate of absorption, comparison between formulation and simulation cannot be made. This non-linearity may be owing to saturable absorption processes (active absorption), induction or inhibition of the metabolism, the first past effect, which is rate/absorption dependent, etc. Those points must be studied before any attempt to establish an IVIVC.

3. Absorption should not be the limiting factor, if the solubility is not the limiting factor in comparison to the drug release, an IVIVC may be attempted. The release must depend on the formulation, and must be the slowest phenomenon vs. dissolution and absorption.

\section{Conclusions}

The pharmaceutical industry has been striving to find a ways to saving precious resources in relevance to the budgets and increasing cost of drug development. IVIVC is a tool applied in various areas and stages of drug development to find a place in the regulatory bodies around the world. IVIVC can serve as surrogate for in vivo bioavailability and to support biowaivers also allows setting the dissolution specification and methods. The substitute of expensive clinical trials with the use of IVIVC is perhaps the most important feature of IVIVC. From the regulatory point of view IVIVC can assist certain scale-up and postapproval changes. IVIVC principles have been mostly applied to oral products, there exists a need to develop methodologies and standards for non-oral delivery systems, to develope more meaningful dissolution and permeation methods.

\section{Acknowledgement}

The authors gratefully acknowledge Mrs. Sangita Sakore (Regulatory affairs, Formulation \& Development, MKPPL, Pune, India) for her contribution.

\section{References}

1. Leeson LJ (1995) In vitrol In vivo correlations. Drug Info J 29: 903-915.

2. US Department of Health and Human Services (1995) Guidance for industry: Immediate release solid oral dosage forms scale-up and postapproval changes: chemistry, manufacturing, and controls, in vitro dissolution testing, and in vivo bioequivalence documentation. Center for drug Evaluation and Research.

3. Center for Drug Evaluation, USFDA (1995) Guidance for Industry: Immediate Release Solid Oral Dosage Forms: Scale Up and Post Approval Changes.

4. Uppoor VRS (2001) Regulatory perspectives on in vitro (dissolution)/in vivo (bioavailability) correlations. J Control Rel 72: 127-132.

5. Jaber E (2006) In vitro - In vivo Correlation: From Theory to Applications. Pharm Pharmaceut Sci 9: 169-189.

6. Food and Drug Administration (1997) Guidance for industry: extended release oral dosage forms: Development, evaluation, and application of in vitro/in vivo correlations

7. Sirisuth N, Eddington ND (2002) In vitro in vivo correlations, systemic methods for the development and validation of an IVIVC metoprolol and naproxen drug examples. Int J Generic Drugs 3: 250-258.

8. Qureshi SA (2010) In Vitro-In Vivo Correlation (IVIVC) and Determining Drug Concentrations in Blood from Dissolution Testing - A Simple and Practical Approach. Open Drug Delivery J 4: 38-47.

9. O'Hara T, Hayes S, Davis J, Devane J, Smart T, et al. (2001) In vivo-In Vitro Correlation (IVIVC) Modeling Incorporating a Convolution Step. J Pharmacokinet Pharmacodyn 28: 277-298.

10. Gaynor C, Dunne A, Davis J (2008) A Differential Equations Approach to In Vitro - In Vivo Correlation Modelling. International Biometric Conference, Dublin.

11. Dunne A (2007) Approaches to developing IVIVC models, Ch 5. In Chilukuri Sunkara and Young (Eds), Pharmaceutical Product Development: In Vitro - In vivo Correlation. Taylor and Francis, New York.

12. Beal SL, Sheiner LB (1992) NONMEM User's Guides, NONMEM Project Group, University of California, San Francisco.

13. Meyer MC, Straughn AB, Mhatre RM, Shah VP, Williams RL (1998) Lack of in vitro / in vivo correlations of $50 \mathrm{mg}$ and $250 \mathrm{mg}$ primidone tablets. Pharm Res 15: $1085-1089$.

14. Food and Drug Administration. Rockville, MD Abdou, HM (1989) Dissolution Bioavailability and Bioequivalence. Easton Pennsylvania: Mack Printing.

15. Buchwald $P$ (2003) Direct, differential-equation-based in-vitro-in-vivo correlation (IVIVC) method. J Pharm Pharmacol 55: 495-504.

16. Dressman JB, Amidon GL, Reppas C, Shah VP (1998) Dissolution testing as a prognostic tool for oral drug absorption: immediate release dosage forms. Pharm Res 15: 11-22.

17. Corrigan OI (1997) The biopharmaceutic drug classification and drugs administered in extended release (ER) formulations. Adv Exp Med Biol 423 111-128.

18. Yu H, Joves $\mathrm{R}$ (2004) The STATs of cancer--new molecular targets come of age. Nat Rev Cancer 4: 97-105

19. Wagner JG, Nelson E (1964) Kinetic analysis of blood levels and urinary excretion in the absorptive phase after single doses of drug. J Pharm Sci 53 : 1392-1403.

20. Loo JC, Riegelman S (1968) New method for calculating the intrinsic absorption rate of drugs. J Pharm Sci 57: 918-928.

21. Cutler DJ (1978) Linear system analysis in pharmacokinetics. Pharmacokine Biopharm 6: 265-282.

22. Brockmeier D, Voegele D, von Hattingberg HM (1983) In vitro-in vivo correlation, a time scaling problem? Basic techniques for testing equivalence. Arzneimittelforschung 33: 598-601.

23. Jayaprakasam B, Seeram NP, Nairs MG (2003) Anticancer and antiinflammatory activities of cucurbitacins from Cucurbita andreana. Cancer Lett 189: 11-16. 
Citation: Sakore S, Chakraborty B (2011) In Vitro-In Vivo Correlation (IVIVC): A Strategic Tool in Drug Development. J Bioequiv Availab S3. doi:10.4172/jbb.S3-001

24. Chilukuri DM, Sunkara G (2003) IVIVC: An Important Tool in the Development of Drug Delivery Systems. Drug Deliv Technol 3: 4

25. Young D, Devane JG, Butler J (1997) In Vitro-In Vivo Correlations. New York: Plenum press.

26. Amidon GL, Robinson JR, Williams RL (1997) Scientific Foundations for Regulating Drug Product Quality. American Association of Pharmaceutical Scientists. Alexandria, Virginia: AAPS Press.

27. Amidon GL, Lennernas H, Shah VP, Crison JR (1995) A theoretical basis for a biopharmaceutic drug classification: The correlation of in vitro drug product dissolution and in vivo bioavailabilty. Pharm Res 12: 413-419.

28. Gohel MC, Mehta NR (2005) An audit of recent inputs on biopharmaceutical classification system. Pharmaceut Rev.

29. Yu LX, Amidon GL, Polli JE, Zhao H, Mehta MU, et al. (2002) Biopharmaceutics classification system: The scientific basis for biowaiver extension. Pharm Res 19: $921-925$

30. Wilding I (1999) Evolution of the Biopharmaceutics Classification System (BCS) to Modified Release (MR) formulations: What do we need to consider? Eur J Pharm Sci 8: 157-159.

31. Devane J (1998) Oral drug delivery technology: Addressing the solubility/ permeability paradigm. Pharmaceut Technol 22: 68-80.

32. Helga M (2002) The Biopharmaceutical Classification System (BCS) and its usage. Drugs Made in Germany 45: 63-65.

33. Dressman J, Butler J, Hempenstall J, Reppas C (2001) The BCS: Where do we go from here? Pharmaceut Technol 25: 68-76.

34. Sievert B, Siewert M (1998) Dissolution tests for ER products. Dissolut Technol 5.
35. Shargel L, Yu ABC (1993) Applied Biopharmaceutics and Pharmacokinetics. East Norwark, Connecticut: Appleton \& Lange.

36. Galia E, Nicolaides E, Horter D, Lobenberg R, Reppas C, et al. (1998) Evaluation of various dissolution media for predicting in vivo performance of class I and class II drugs. Pharm Res 15: 698-705.

37. Abdou HM (1989) Dissolution, Bioavailability and Bioequivalence. Easton, Pennsylvania: Mack Printing.

38. Strickley RG (1999) Parenteral formulations of small molecules therapeutics marketed in the United States Part I, PDA. J Pharm Sci Tech 53: 324-349.

39. Modi NB, Lam A, Lindemulder E, Wang B, Gupta SK (2000) Application of in vitro-in vivo correlation (IVIVC) in setting formulation release specifications. Biopharm Drug Dispos 21: 321-326.

40. Venkatesh S, Lipper RA (2000) Role of the development scientist in compound lead selection and optimisation. J Pharm Sci 89: 145-154.

41. Hwang SS, Gorsline JJ, Louie J, Dye D, Guinta D, et al. (1995) in vitro and in vivo evaluation of a once-daily controlled release pseudoephedrine product. J Clin Pharmacol 35: 259-267.

42. FDA (1997) Guidance for Industry: SUPAC-MR: Modified release solid oral dosage forms: scale-up and post-approval changes: chemistry, manufacturing and controls, in vitro dissolution testing, and in vivo bioequivalence documentation.

43. Young D, Chilukuri D, Becker R, Bigora S, Farrell C, et al. (2002) Approaches to developing a Level-A IVIVC for injectable dosage forms. AAPS Pharm Sci 4: M1357. 